\title{
Oscillations of tori in the pseudo-Newtonian potential
}

\author{
E. Šrámková ${ }^{1}$, U. Torkelsson ${ }^{2}$, and M. A. Abramowicz ${ }^{2,3}$ \\ 1 Institute of Physics, Silesian University in Opava, Bezručovo nám 13, 74601 Opava, Czech Republic \\ e-mail: sram_eva@centrum.cz \\ 2 Department of Physics, Göteborg University, 41296 Göteborg, Sweden \\ 3 Copernicus Astronomical Centre, Bartycka 18, 00-716 Warszawa, Poland
}

Received 5 July 2006 / Accepted 21 February 2007

ABSTRACT

\begin{abstract}
Context. The high-frequency quasi-periodic oscillations (HF QPOs) in neutron star and stellar-mass black hole X-ray binaries may be the result of a resonance between the radial and vertical epicyclic oscillations in strong gravity.

Aims. In this paper we investigate the resonant coupling between the epicyclic modes in a torus in a strong gravitational field.

Methods. We perform numerical simulations of axisymmetric constant angular momentum tori in the pseudo-Newtonian potential. The epicyclic motion is excited by adding a constant radial velocity to the torus.

Results. We verify that slender tori perform epicyclic motions at the frequencies of free particles, but the epicyclic frequencies decrease as the tori grow thicker. More importantly, and in contrast to previous numerical studies, we do not find a coupling between the radial and vertical epicyclic motions. The appearance of other modes than the radial epicyclic motion in our simulations is rather due to small numerical deviations from exact equilibrium in the initial state of our torus.

Conclusions. We find that there is no pressure coupling between the two axisymmetric epicyclic modes as long as the torus is symmetric with respect to the equatorial plane. However we also find that there are other modes in the disc that may be more attractive for explaining the HF QPOs.
\end{abstract}

Key words. accretion: accretion disks - black hole physics

\section{Introduction}

Observations by Rossi-XTE have revealed that some accreting neutron stars (NS) and stellar-mass black holes (BH) show quasi-periodic oscillations in their X-ray fluxes with frequencies of $100-1300 \mathrm{~Hz}$ (see van der Klis 2000 , for a review). Some $\mathrm{X}$-ray sources are simultaneously varying on two frequencies that form a ratio of 3:2. For BH QPOs this was first noticed by Abramowicz \& Kluźniak (2001) (see also Strohmayer 2001; McClintock \& Remillard 2006). Abramowicz et al. (2003) argued that this is also the case for NS QPOs: although the ratio is varying, it clearly distinguishes the same 3:2 value (see Belloni et al. 2005 for a criticism). The mechanism that is responsible for this phenomenon is not fully understood. It has been pointed out by Kluźniak \& Abramowicz (2000) that the rational frequency ratio could be formed naturally in a resonance between two modes of accretion disc oscillations. Radial and vertical epicyclic oscillations in an accretion disc have been the most often discussed possibility (e.g. Abramowicz \& Kluźniak 2004, or a collection of review articles in Abramowicz 2005). In Newtonian gravity with the $1 / r$ potential the epicyclic frequencies $\omega_{\mathrm{R}}$ and $\omega_{z}$ are both equal to the Keplerian frequency $\Omega_{\mathrm{K}}$. This is no longer the case in the strong gravitational field close to a compact object, where $\omega_{z}>\omega_{\mathrm{R}}$, which allows the two frequencies to assume a 3:2 commensurability at some location.

Several authors have numerically studied oscillations of thick accretion discs around black holes and neutron stars in the QPO context in both the Newtonian (Rubio-Herrera \& Lee 2005a,b) and the relativistic regime (Rezzolla et al. 2003a,b; Montero et al. 2004; Zanotti et al. 2005). Recently, Lee et al. (2004) have performed numerical simulations of pseudoNewtonian slender tori in order to study the response of the torus to impulsive and periodic perturbations associated with the spin of the neutron star. They reported that a vertical epicyclic motion was excited in the torus due to the non-linear coupling between the epicyclic modes when the applied perturbation was purely radial, and found its response to a periodic perturbation to be dependent on the difference between the spin frequency and the frequency difference between the two epicyclic modes (see also Kluźniak et al. 2004; Lee et al. 2004).

In this paper we present a numerical study of oscillatory modes of slender tori in equilibrium that are given a small impulsive radial perturbation. We start in Sect. 2 by writing down the hydrodynamic equations, from which we construct our equilibrium tori, and then we describe the numerical method that we use to follow the time evolution of the tori. The results of our numerical simulations are described in Sect. 3, while Sect. 4 is devoted to a discussion of the results and our conclusions.

\section{Model}

\subsection{Hydrodynamics}

We use the equations of nonrelativistic ideal hydrodynamics to describe the oscillations of a torus around a black hole. Ignoring the effects of radiative transport and self-gravity, the equations take the form

$$
\begin{aligned}
\frac{\partial \rho}{\partial t}+\nabla \cdot(\rho \boldsymbol{v}) & =0, \\
\rho \frac{\partial \boldsymbol{v}}{\partial t}+(\rho \boldsymbol{v} \cdot \boldsymbol{\nabla}) \boldsymbol{v} & =-\nabla P-\rho \nabla \Phi, \\
\frac{\partial(\rho \epsilon)}{\partial t}+\nabla \cdot(\rho \boldsymbol{\epsilon}) & =-P \boldsymbol{\nabla} \cdot \boldsymbol{v},
\end{aligned}
$$




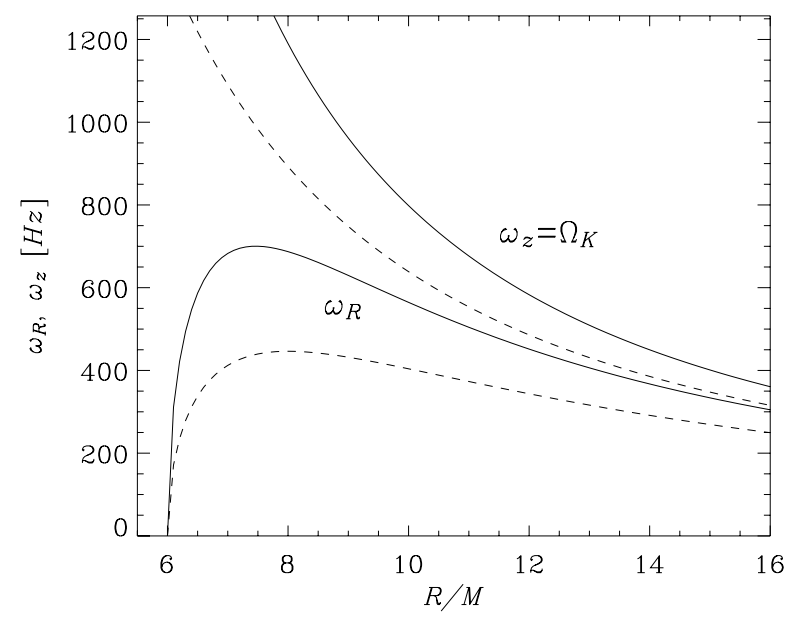

Fig. 1. Radial $\left(\omega_{\mathrm{R}}\right)$ and vertical $\left(\omega_{z}=\Omega_{\mathrm{K}}\right)$ epicyclic frequencies in the Paczyński and Wiita potential, and the corresponding radial (lower dashed curve) and vertical (upper dashed curve) epicyclic frequencies in the Schwarzschild metric, for a $M=10 M_{\odot}$ black hole.

where $\rho$ and $\epsilon$ are the density of mass and the specific internal energy, $v$ is the fluid velocity, $\Phi$ the gravitational potential and $P$ is the pressure, which is given by the equation of state for an ideal gas, $P=\rho \epsilon(\gamma-1)$, with $\gamma=4 / 3$.

The most important general relativistic effects in the Schwarzschild metric are mimicked by using the pseudoNewtonian potential (Paczyński \& Wiita 1980)

$\Phi=-\frac{G M}{\left(r-r_{\mathrm{g}}\right)}$,

where $r$ is the spherical radius and $r_{\mathrm{g}}=2 G M / c^{2}$ is the Schwarzschild radius of the black hole. In this potential, the epicyclic frequencies have qualitatively the same behaviour as in the Schwarzschild spacetime, i.e. $\omega_{\mathrm{R}}<\omega_{z}=\Omega_{\mathrm{K}}$ (see Fig. 1), and are explicitly given by $\omega_{\mathrm{R}}=2 \pi \nu_{\mathrm{R}}=$ $\sqrt{G M\left(R-3 r_{\mathrm{g}}\right)} / \sqrt{R\left(R-r_{\mathrm{g}}\right)^{3}}$ and $\omega_{z}=2 \pi v_{z}=\sqrt{G M / R} /\left(R-r_{\mathrm{g}}\right)$. In the Schwarzschild metric, the frequencies assume the 3:2 ratio at $R=10.8 G M / c^{2}$, while in the Paczyński \& Wiita potential this resonance occurs at $R=9.2 \mathrm{GM} / \mathrm{c}^{2}$.

We construct a non-accreting torus in hydrostatic equilibrium with constant specific angular momentum $l=l_{0}$ as our initial state. For convenience we will adopt geometric units, where $G=c=1$, for the rest of the paper. The only non-zero velocity component is then the azimuthal component, which in cylindrical coordinates $\{R, \phi, z\}$ is given by $v_{\phi}=\Omega R$. The equilibrium configuration is then governed by the time-independent Euler equation

$-\frac{1}{\rho} \nabla P=\nabla \Phi-\Omega^{2} \boldsymbol{R}$.

For a barotropic fluid, in which $\Omega=\Omega(R)$, we can define a rotational potential $\Phi_{\text {rot }}=\int \Omega^{2} R \mathrm{~d} R=\int l_{0}^{2} / R^{3} \mathrm{~d} R$. In addition we use a polytropic equation of state, $P=K \rho^{\gamma}$, where $K$ denotes the polytropic constant. The Euler equation can then be integrated to give an expression for the equipotentials $W$ that determine the shape of the torus:

$\frac{\gamma}{(\gamma-1)} \frac{P}{\rho}+\Phi_{\mathrm{eff}}+C=W=$ const.

Here $\Phi_{\text {eff }}=\Phi+\Phi_{\text {rot }}$ is the effective potential and $C$ an integration constant defining the surface of the torus. For particular values of

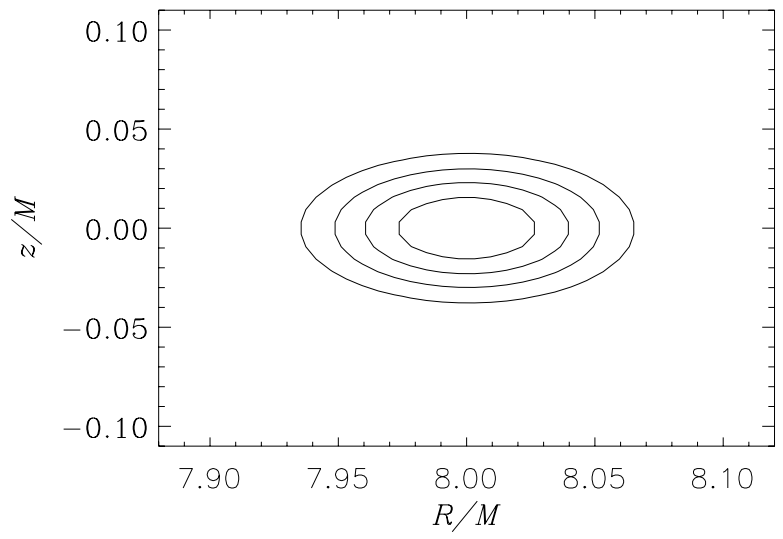

Fig. 2. Density contours in a meridional cross-section of a slender torus at $R=8 M$ with radial extent of approximately $0.15 M$.

the specific angular momentum, an equilibrium torus can now be constructed by filling the appropriate equipotential surfaces with a rotating fluid. An example of the meridional cross-section of a slender (in the sense that its radial extent is much smaller than its distance from the central object) torus is shown in Fig. 2.

\subsection{Numerical simulations}

The numerical simulations in this paper are performed using the two-dimensional ZEUS-2D code by Stone \& Norman (1992) that solves Eqs. (1)-(3). ZEUS is a time-explicit, Eulerian finite difference code that uses a staggered grid. Shock waves are handled by an artificial viscosity tensor. The magnetic field, selfgravity and radiation transport in ZEUS were switched off during our simulations.

We study two different groups of models (Table 1). Models A1-A4 represent slender tori with a radial extent of approximately $0.15 \mathrm{M}$ at different radial positions (see Fig. 2). Models B1-B4 make up a series of thicker (non-slender) tori. We perturb the tori by adding a velocity field that is constant in space at $t=0$. The perturbation is always subsonic, corresponding to a Mach number at the torus center of mass of 0.3 .

We use a uniform grid in cylindrical coordinates in all our simulations and the resolutions of the different models are specified in Table 1.

\section{Results}

For the data analysis we look at some of the characteristic quantities as functions of time, such as the total kinetic energy, the mean of the square of the density (as was previously done by e.g. Zanotti et al. 2005; and Blaes et al. 2006a), and (following Lee et al. 2004) the radial and vertical positions of the centre of mass of the torus, as well as snapshots of the density, internal energy and velocity field inside the torus.

\subsection{Slender torus oscillations}

We consider a slender torus (models A1-A4) and perturb it slightly by adding a purely radial constant velocity field at $t=0$. The torus then performs radial oscillations at the radial epicyclic frequency of a test particle in a circular orbit at the centre of the torus (Fig. 3). We also find a small oscillation in the vertical direction at the vertical epicyclic frequency of a test particle (see Table 1 for the frequencies measured from the simulations), but 
Table 1. Properties of the models. From left to right the columns give the name of the model, grid resolution, radial ( $R$-grid) and vertical ( $z$-grid) boundaries of the grid, the inner $\left(R_{\mathrm{in}}\right)$, central $\left(R_{\mathrm{c}}\right)$, and outer $\left(R_{\text {out }}\right)$ radii, the initial velocity kick in terms of the Mach number $M$ at the centre of the torus, and radial $\omega_{\mathrm{R}}$ and vertical $\omega_{z}$ epicyclic frequencies of the torus centre in units of $\Omega_{\mathrm{K}}$.

\begin{tabular}{cccccccccc}
\hline \hline Model & Grid points in $(R, z)$ & $R$-grid & $z$-grid & $R_{\text {in }} / M$ & $R_{\mathrm{c}} / M$ & $R_{\text {out }} / M$ & $M$ & $\omega_{\mathrm{R}} / \Omega_{\mathrm{K}}$ & $\omega_{z} / \Omega_{\mathrm{K}}$ \\
\hline A1 & $128 \times 128$ & $6.95-7.65$ & $-0.35-0.35$ & 7.25 & 7.33 & 7.41 & 0.3 & 0.50 & 1.00 \\
A2 & $128 \times 128$ & $7.65-8.35$ & $-0.35-0.35$ & 7.92 & 8.00 & 8.08 & 0.3 & 0.58 & 1.00 \\
A3 & $128 \times 128$ & $8.80-9.60$ & $-0.35-0.35$ & 9.12 & 9.20 & 9.28 & 0.3 & 0.67 & 1.00 \\
A4 & $200 \times 200$ & $8.80-9.60$ & $-0.35-0.35$ & 9.12 & 9.20 & 9.28 & 0.3 & 0.67 & 1.00 \\
\hline B1 & $128 \times 128$ & $8.80-09.50$ & $-0.40-0.40$ & 9.100 & 9.2 & 09.302 & 0.3 & 0.67 & 0.99 \\
B2 & $156 \times 156$ & $7.00-12.00$ & $-2.50-2.50$ & 7.800 & 9.2 & 11.069 & 0.3 & 0.64 & 0.93 \\
B3 & $186 \times 186$ & $6.00-15.00$ & $-4.50-4.50$ & 6.999 & 9.2 & 13.998 & 0.3 & 0.57 & 0.76 \\
B4 & $200 \times 200$ & $5.50-17.00$ & $-5.75-5.75$ & 6.000 & 9.2 & 16.503 & 0.3 & 0.50 & 0.60 \\
\hline
\end{tabular}
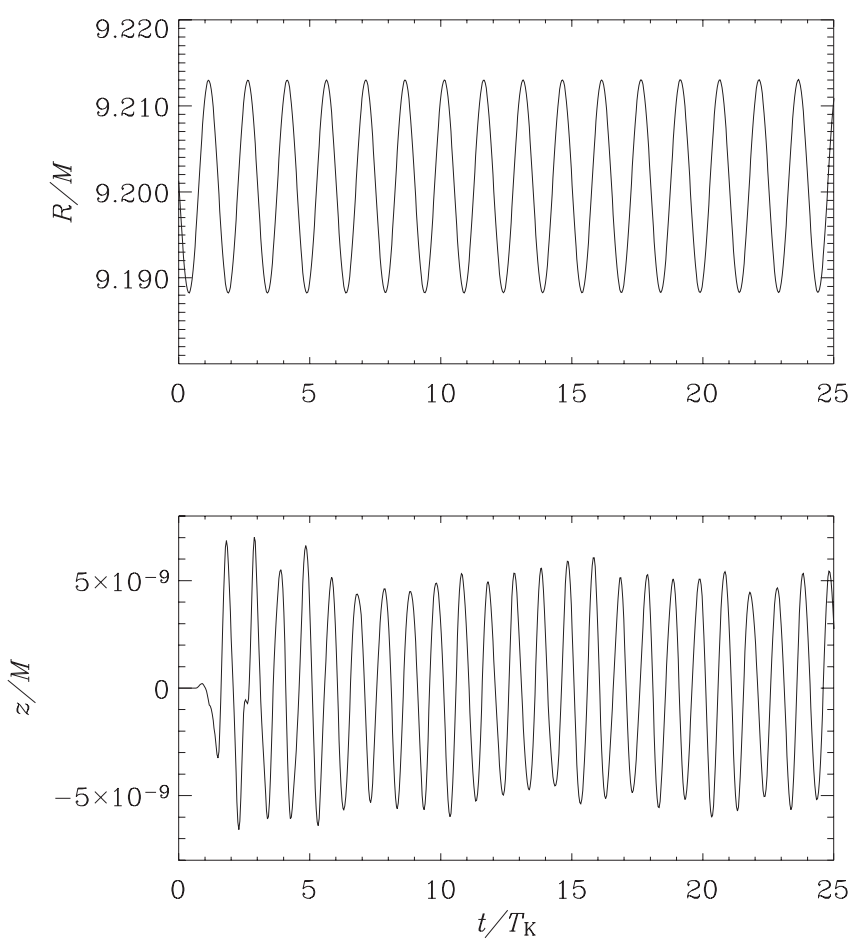

Fig. 3. Radial (top) and vertical (bottom) oscillations of a torus centered at $R=9.2 M$ (model A3) where the two epicyclic modes are in a 3:2 ratio. The time is given in units of the Keplerian period $T_{\mathrm{K}}$ defined at the centre of the torus. The torus performs a radial oscillation with an amplitude of $0.01 \mathrm{M}$. The vertical oscillation excited here is seven orders of magnitude smaller than the radial oscillation.

the amplitude of this motion is more than six orders of magnitude smaller than that of the radial epicyclic motion. The small amplitude of the vertical oscillation is independent of both the amplitude of the radial epicyclic motion and the radial position of the centre of the torus. Moreover, it decreases with increasing numerical resolution, as shown in Fig. 4. Our conclusion is therefore that it is not the result of a resonance between the two epicyclic modes. Rather it is excited by numerical noise in the simulations.

The power spectra of the mean of the square of the density and the total vertical kinetic energy for model A 2 can be seen in Figs. 5 and 6 . The $\left\langle\rho^{2}\right\rangle$ power spectrum is not sensitive to incompressible modes, such as the vertical epicyclic mode, but the radial epicyclic mode is weakly compressible because of the cylindrical geometry, and thus we can see it in Fig. 5. It corresponds to the left peak at $\omega_{\mathrm{R}}=0.57 \Omega_{\mathrm{K}}$. Beyond that, there are two other peaks in the power spectrum of $\left\langle\rho^{2}\right\rangle$. These peaks appear independently of whether the torus has been perturbed or

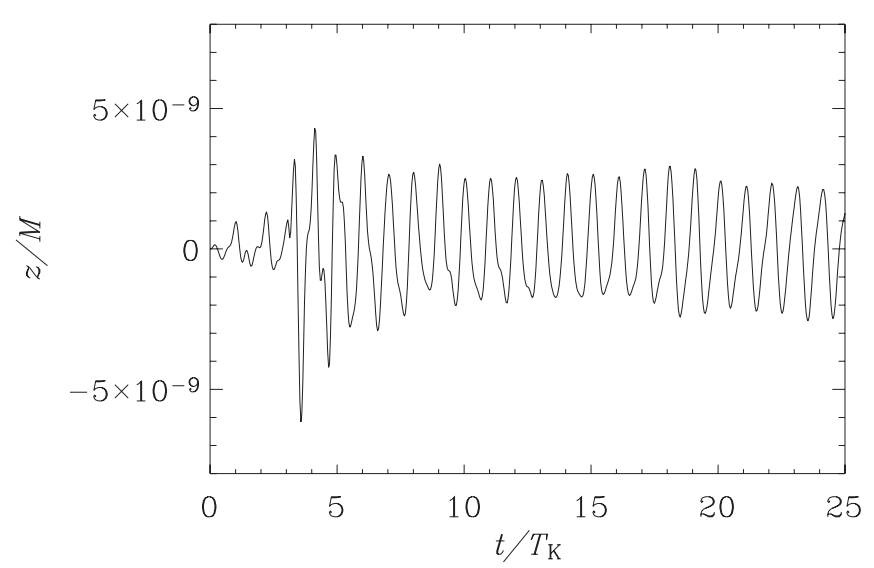

Fig. 4. Same as the bottom panel in Fig. 3, but using a finer grid (model A4). Note the decrease of the amplitude of the oscillation compared to Fig. 3.

not (compare the top and bottom panels of Fig. 5). The strong peak at $1.5 \Omega_{\mathrm{K}}$ is present in the spectrum at this frequency regardless of the location of the torus, while the less prominent peak at approximately $0.86 \Omega_{\mathrm{K}}$ slightly changes its position depending on the location of the torus.

Blaes et al. (2006a) have calculated the lowest-order slender torus modes. Two of these modes, the incompressible "plusmode" and the acoustic "breathing" mode have frequencies of $0.87 \Omega_{\mathrm{K}}$ and $1.54 \Omega_{\mathrm{K}}$ for the torus of model A2 (see their Table 2 for explicit expressions). These frequencies match well with the peaks at $0.86 \Omega_{\mathrm{K}}$ and $1.5 \Omega_{\mathrm{K}}$ in Fig. 5 .

The $1.5 \Omega_{\mathrm{K}}$ peak also appears in the power spectrum of the total vertical kinetic energy, as seen in Fig. 6. We also find four minor peaks in the power spectrum of the vertical kinetic energy at $0.45 \Omega_{\mathrm{K}}, 0.75 \Omega_{\mathrm{K}}, 1.05 \Omega_{\mathrm{K}}$, and $1.2 \Omega_{\mathrm{K}}$. The peaks at $0.45 \Omega_{\mathrm{K}}$ and $1.05 \Omega_{\mathrm{K}}$ are absent in the case of an unperturbed torus (bottom panel of Fig. 6). None of the minor peaks have any corresponding peaks in the power spectrum of $\left\langle\rho^{2}\right\rangle$, and they cannot be identified with any of the slender torus modes that Blaes et al. (2006a) calculated. An intriguing feature of Fig. 6 is the lack of power at the frequency of the radial epicyclic mode, which suggests that this mode has a negative influence on the vertical motion.

Figure 7 shows four snapshots of $\rho \boldsymbol{v}$ inside the torus (model A2) after substraction of the mean radial motion of the torus. They cover one period of the breathing mode $2 \pi /\left(1.54 \Omega_{\mathrm{K}}\right)$. The velocity pattern is dominated by the breathing mode, though we do see radial flows that match the plus-mode too. 

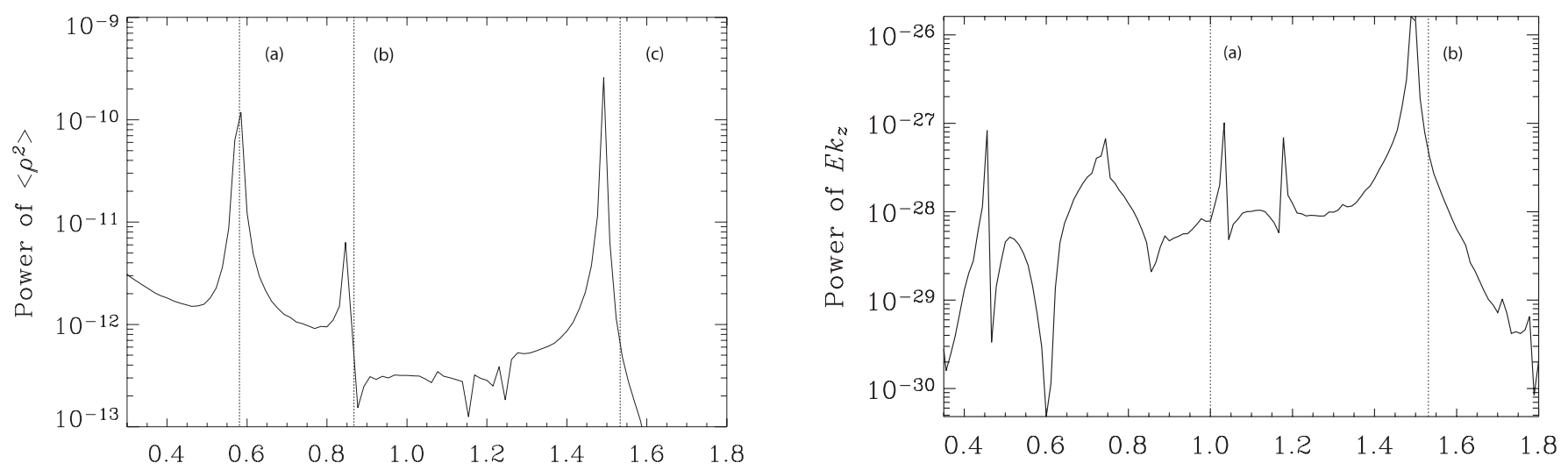

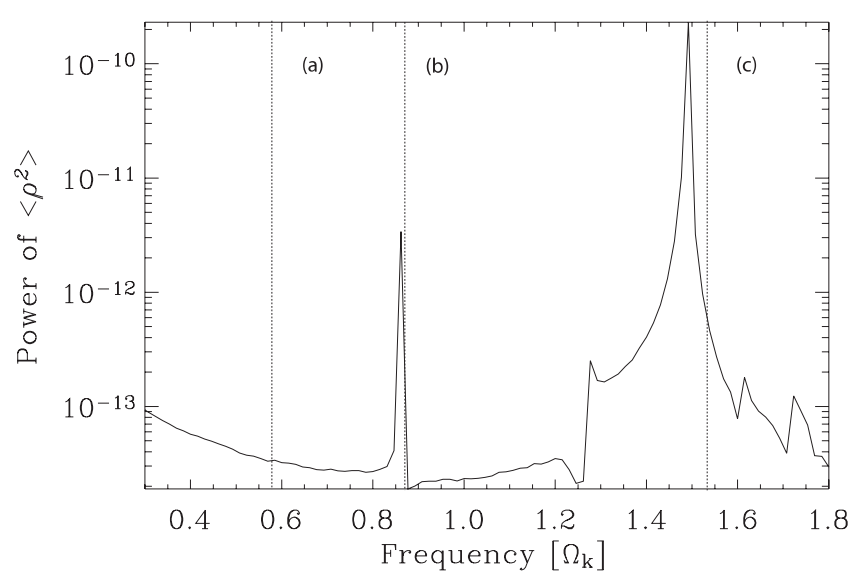

Fig. 5. Top: power spectrum of $\left\langle\rho^{2}\right\rangle$ of a radially perturbed slender torus (model A2). Frequencies are given in units of $\Omega_{\mathrm{K}}$, the Keplerian angular velocity at the centre of the torus. The left peak at approximately $0.58 \Omega_{\mathrm{K}}$ corresponds to the radial epicyclic motion (see Table 1). The other main peaks are located at about $0.86 \Omega_{\mathrm{K}}$ and $1.5 \Omega_{\mathrm{K}}$. The vertical lines denote the frequencies of the radial epicyclic mode (a), the plus-mode (b) and the breathing mode (c). Bottom: same as the top panel, but for an unperturbed torus. The peak belonging to the radial motion is absent, but the other two peaks remain with approximately the same power, as in the top panel.

\subsection{Epicyclic oscillations in thick tori}

In order to excite both the epicyclic modes, we applied a perturbation in both the radial and the vertical directions. The resulting epicyclic frequencies for four different tori (models B1-B4) are presented in Table 1. For an infinitely slender torus, the frequencies are consistent with the epicyclic frequencies of a test particle at the torus centre, $\omega_{\mathrm{R}}=0.666 \Omega_{\mathrm{K}}$ and $\omega_{z}=\Omega_{\mathrm{K}}$. The frequencies decrease as the torus grows thicker, as seen from Table 1 and Fig. 8. A similar trend was reported by Rubio-Herrera \& Lee (2005b), and Blaes et al. (2006b), where it has been explored analytically for slightly non-slender Newtonian tori. It is worth mentioning that even when we excite both the epicyclic modes, we do not see any exchange of energy between them (see Fig. 9).

\section{Discussion and conclusions}

\subsection{Epicyclic oscillations}

We have performed two-dimensional numerical simulations of oscillations of constant angular momentum tori in the Paczyński and Wiita potential. The tori were given an impulsive radial

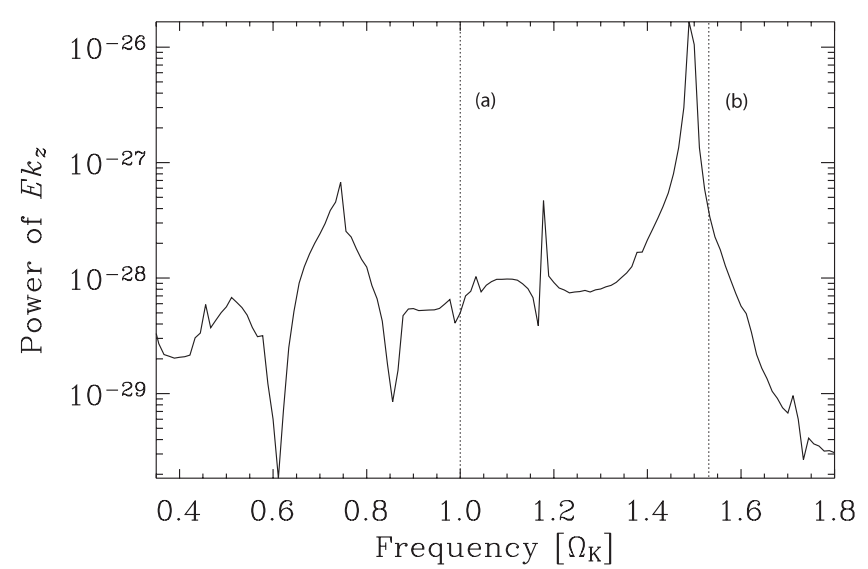

Fig. 6. Top: power spectrum of the total vertical kinetic energy of a radially perturbed slender torus centered at $R=8 M$ (model A2). Frequencies are given in units of $\Omega_{\mathrm{K}}$. Note the major peak at $1.5 \Omega_{\mathrm{K}}$ and the smaller peaks at $0.45 \Omega_{\mathrm{K}}, 0.75 \Omega_{\mathrm{K}}, 1.05 \Omega_{\mathrm{K}}$, and $1.2 \Omega_{\mathrm{K}}$. The vertical lines denote the frequencies of the vertical epicyclic mode (a) and the breathing mode (b) calculated for this model. Bottom: same as the top panel, but for an unperturbed torus. The $0.45 \Omega_{\mathrm{K}}$ and $1.05 \Omega_{\mathrm{K}}$ peaks are missing in this case.

perturbation and were allowed to evolve in time afterwards. The tori are then describing radial epicyclic motions. For a sufficiently slender torus the frequency of this oscillation agrees with that of a test particle. As the torus grows thicker we find that the epicyclic frequencies significantly decrease. This is in accord with previous numerical results by Rubio-Herrera \& Lee (2005b), as well as recent analytic work where the expressions for the (negative) corrections to the epicyclic frequencies were derived using a perturbative method accurate to second-order in the extent of the torus (Blaes et al. 2006b).

One of the main objectives of this work is to investigate the modes that may be excited by a radial epicyclic oscillation in the torus. In particular, we look for the resonant coupling between the radial and the vertical epicyclic modes, which Kluźniak and Abramowicz suggested plays a significant role in the high-frequency QPOs. We find that the centre of mass of the torus performs a vertical oscillation of a very low amplitude, but it is independent of the amplitude of the radial epicyclic oscillation and the location of the torus, unlike what one would expect if it was due to a resonance between the two epicyclic modes. Rather it is likely that the vertical epicyclic oscillation is excited by numerical noise in the simulations. A coupling between the radial and vertical epicyclic modes in the 

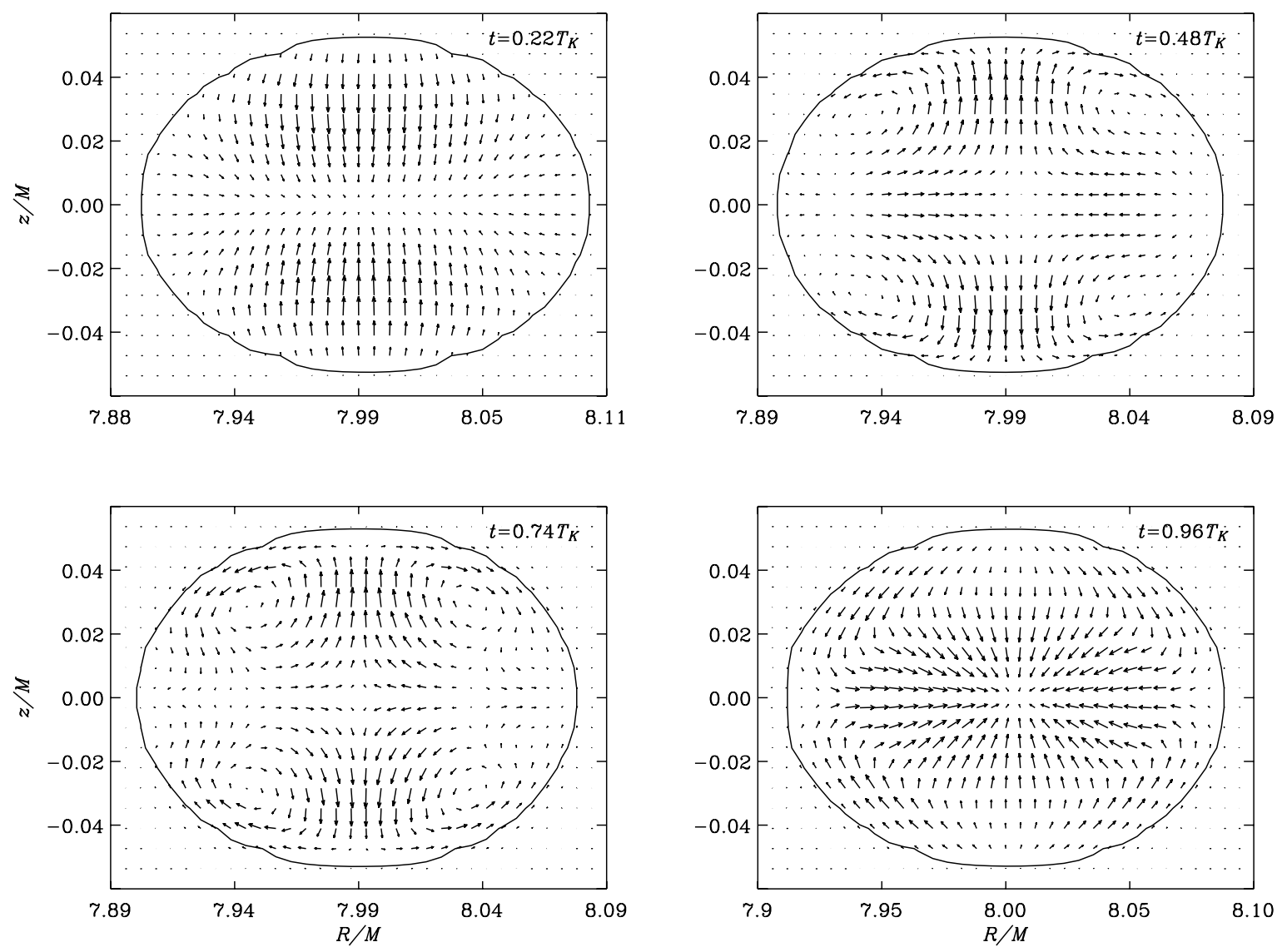

Fig. 7. Snapshots of the $\rho \boldsymbol{v}$ inside the torus (model A2) after substraction of the radial epicyclic motion. The snapshots cover a period of the breathing mode, $T=(1 / 1.54) T_{\mathrm{K}}$, where $T_{\mathrm{K}}$ again denotes the Keplerian period at the centre of the torus. The residual $\rho \boldsymbol{v}$ resembles that of the breathing mode (see Fig. 1 in Blaes et al. 2006a).

3:2 parametric resonance may only happen in a limited range of the parameter space, the Arnold tongue (see e.g. Arnold 1978, 1983). This range is not known a priori, but one relevant parameter is the initial vertical amplitude.

Lee et al. (2004) found that a small vertical epicyclic motion was excited by the radial epicyclic motion in their simulations of an axisymmetric torus. We believe that the reason for this is that their initial torus was not perfectly symmetric around the $z=0$ plane, and thus the initial amplitude of the vertical oscillation was not zero. We did not find that any vertical oscillations were excited in our simulations, in which the initial amplitude of the vertical motion was zero - thus in our case there was not any coupling between the radial and vertical epicyclic modes. This is hardly surprising: conservation of momentum prohibits the excitation of a vertical epicyclic motion of the centre of the torus, if it is initially at rest in the equatorial plane, and no external vertical forces are at play.

In order to find the Arnold tongue empirically, one should perform many simulations similar to these described in our paper, but with different initial vertical amplitudes, and with different ratios (close to $3: 2$ ) of the radial and vertical epicyclic frequencies. This will give the range in the parameter space in which the mode coupling for axisymmetric oscillations may occur.

A more promising approach might be to consider nonaxisymmetric oscillations. The simplest example of nonaxisymmetric vertical motion is an $m=1$ precessing warp in an accretion disc. Such a warp will resonantly drive a radial epicyclic motion (Papaloizou \& Pringle 1983; Papaloizou \& Lin 1995).

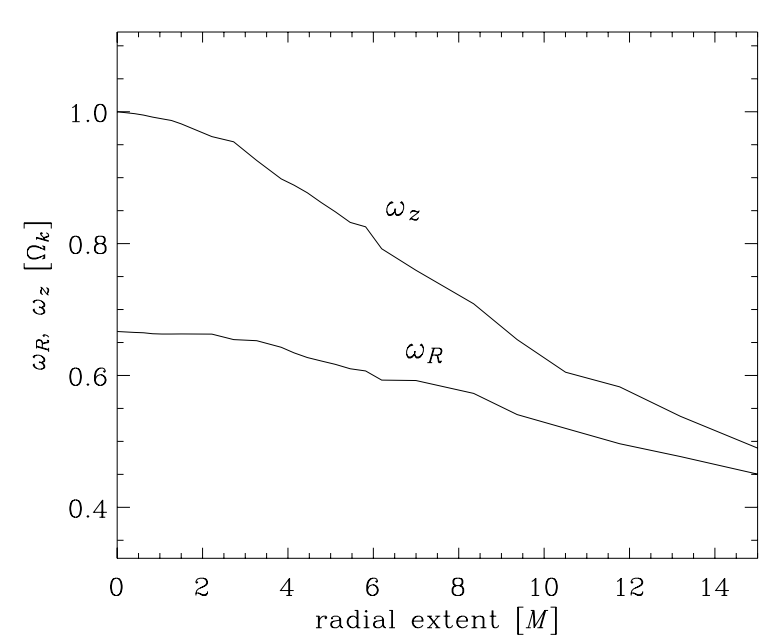

Fig. 8. Radial $\omega_{\mathrm{R}}$ and vertical $\omega_{z}$ epicyclic frequencies of the torus as a function of its width assuming that the torus is centered at $9.2 \mathrm{M}$.

A warp can be produced, for instance, by the radiation pressure from a central radiation source (Pringle 1996). The role of $m=1$ warps for the HF QPOs has been discussed by Kato (2004, 2005).

Another possibility is that the two modes can be coupled by the local fluctuations that are generated by the turbulence in the disc (note that the turbulence is nonaxisymmetric). It is today widely accepted that accretion discs become turbulent because of the magnetorotational instability, that was introduced 

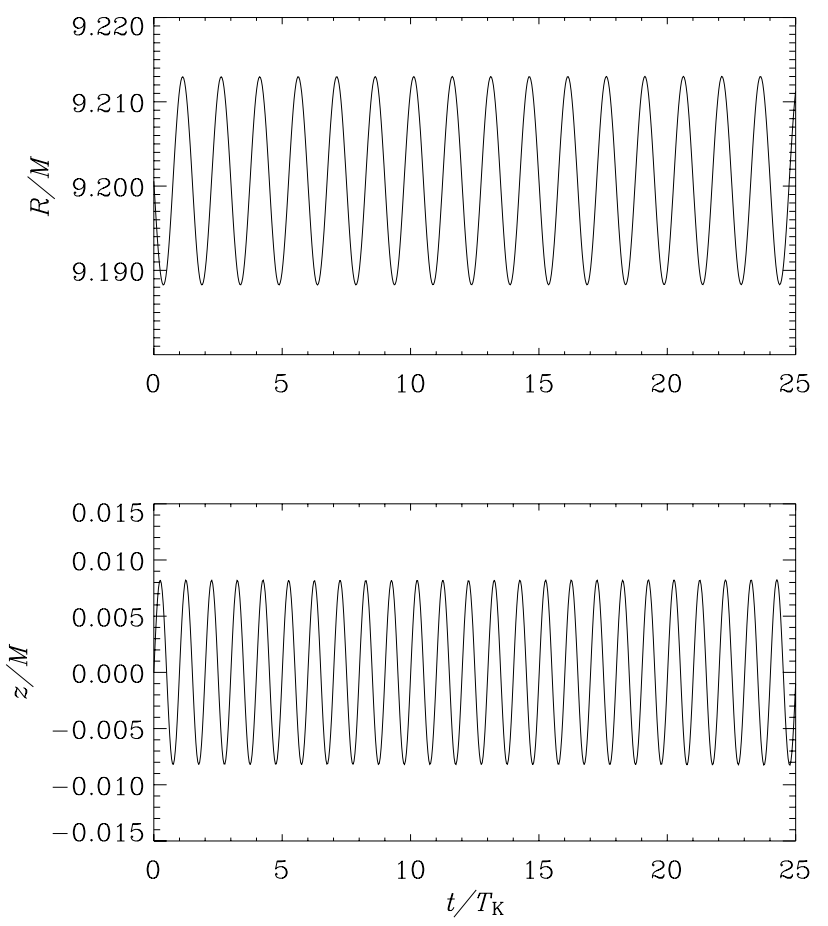

Fig. 9. Radial (top) and vertical (bottom) oscillations of a torus centered at $R=9.2 M$ (model A3) when both the modes are excited. Note that there is no exchange of energy between the two modes.

by Balbus \& Hawley (1991). This turbulence generates the radial transport of angular momentum that drives the accretion and heats the disc, but its role in other aspects of the dynamics of accretion discs has hardly been explored though. Vio et al. (2006) have recently studied how stochastic processes can produce resonances between the epicyclic modes in an almost Keplerian disc.

\subsection{Other oscillatory modes}

A problem with attributing the HF QPOs to a resonance between the two epicyclic modes is that they only occur at a special resonance radius, and thus require a certain amount of finetuning. However our tori contain more oscillatory hydrodynamical modes, and the power spectra of $\left\langle\rho^{2}\right\rangle$ reveal two significant peaks, both of whom occur regardless of whether there is an initial perturbation ${ }^{1}$. Firstly, there is a peak at $1.5 \Omega_{\mathrm{K}}$, which is also displayed in the power spectrum of the vertical energy. This peak appears at approximately the same frequency in all the simulations, no matter what is the location of the torus. It is of interest since its frequency forms a ratio of 3:2 with the Keplerian frequency $\Omega_{\mathrm{K}}$, which is also the vertical epicyclic frequency. It can perhaps be identified as the lowest-order acoustic wave mode (see Blaes et al. 2006a), which has a frequency close to $1.5 \Omega_{\mathrm{K}}$, but with some dependence on the radial location of the torus. It was suggested by Blaes et al. (2006a) that this mode and the vertical epicyclic mode might be responsible for the 3:2 commensurability observed in the HF QPOs.

The other peak at $0.86 \Omega_{\mathrm{K}}$ agrees well with the frequency of a surface gravity wave mode that Blaes and his collaborators labeled as the plus-mode. This oscillatory mode was found to be excited in previous numerical studies with either

1 These modes are excited in our simulations because the equilibrium torus of Sect. 2 is not any longer in perfect equilibrium when it is implemented on our grid. localized (Rubio-Herrera \& Lee 2005a,b), or global (Rezzolla et al. 2003a,b; Montero et al. 2004; and Zanotti et al. 2005) external perturbations, where it was attributed to one of the set of acoustic p-modes of oscillations. Such identification was based on numerical calculations of eigenfunctions and eigenfrequencies of vertically integrated tori, carried out by Rezzolla et al. 2003b, and it was clarified in Blaes et al. (2006a) (see their discussions in Sects. 4.4 and 5.1) that this in general incompressible mode may indeed be related to an acoustic p-mode in the two-dimensional case of a vertically integrated torus. The frequencies of the p-modes were found to appear in an approximate harmonic sequence 2:3:4..., in which the first frequency is consistent with the radial epicyclic frequency of the torus, the second is the plus-mode frequency, and others correspond to the higher-order p-mode frequencies. The plus-mode and the radial epicyclic mode that appear in a 3:2 ratio were proposed by Rezzolla et al. (2003a) as an explanation of the black hole HF QPOs.

Acknowledgements. The authors would like to thank William H. Lee for valuable discussions on his simulations. Most of this work was done during the visit of E.S. to Göteborg University within the SOCRATES-ERASMUS program. We also gratefully acknowledge the hospitality of NORDITA in Copenhagen and Copernicus Astronomical Centre in Warsaw. M.A.A. was supported by the Swedish Research Council, the Polish grant N203 009 31/1466, and the Czech grant MSM 4781305903. E.S. was supported by the Czech grant LC 06014.

\section{References}

Abramowicz, M. A., \& Kluźniak, W. 2001, A\&A, 374, L19

Abramowicz, M. A., \& Kluźniak, W. 2004, AIP Conf. Proc., 714, 21

Abramowicz, M. A., Bulik, T., Bursa, M., \& Kluźniak, W. 2003, A\&A, 404, L21 Abramowicz, M. A. (ed.) 2005, Nordita Workdays on QPOs, Astron. Nachr., 326(9)

Arnold, V. I. 1978, Mathematical methods of classical mechanics (New York: Springer)

Arnold, V. I. 1983, Geometrical methods in the theory of ordinary differential equations, in Grundlehren der Mathematischen Wissenschaften (New York: Springer)

Balbus, S. A., \& Hawley, J. F. 1991, ApJ, 376, 214

Belloni, T., Méndez, M., \& Homan, J. 2005, A\&A, 437, 209

Blaes, O. 1985, MNRAS, 216, 553

Blaes, O. M., Arras, P., \& Fragile, C. P. 2006a, MNRAS, 369, 1235

Blaes, O. M., Šrámková, E., Abramowicz, M. A., Kluźniak, W., \& Torkelsson, U. 2006b, ApJ, submitted

Kato, S. 2004, PASJ, 56, 905

Kato, S. 2005, PASJ, 57, 699

van der Klis, M. 2000, Ann. Rev. Astr. Ap., 38, 717

Kluźniak, W., \& Abramowicz, M. A. 2000, Phys. Rev. Lett., submitted [arXiv: astro-ph/0105057]

Kluźniak, W., Abramowicz, M. A., Kato, S., Lee, W. H., \& Stergioulas, N. 2004, ApJ, 603, L89

Lee, W. H., Abramowicz, M. A., \& Kluźniak, W. 2004, ApJ, 603, L93

McClintock, J. E., \& Remillard, R. A. 2006, in Compact Stellar X-ray Sources, ed. W. H. G. Lewin, \& M. van der Klis (Cambridge: Cam. Univ. Press), 157 Montero, P. J., Rezzolla, L., \& Yoshida, S. 2004, MNRAS, 354, 1040

Paczyński, B., \& Wiita, P. J. 1980, A\&A, 88, 23

Papaloizou, J. C. B., \& Lin, D. N. C. 1995, ApJ, 438, 841

Papaloizou, J. C. B., \& Pringle, J. E. 1983, MNRAS, 202, 1181

Pringle, J. E. 1996, MNRAS, 281, 357

Rezzolla, L., Yoshida, S., Maccarone, T. J., \& Zanotti, O. 2003a, MNRAS, 344, L37

Rezzolla, L., Yoshida, S., \& Zanotti, O. 2003b, MNRAS, 344, 978

Rubio-Herrera, E., \& Lee, W. H. 2005a, MNRAS, 357, L31

Rubio-Herrera, E., \& Lee, W. H. 2005b, MNRAS, 362, 789-798

Stone, J. M., \& Norman, M. L. 1992, ApJS, 80, 791

Strohmayer, T. 2001, ApJ, 554, L169

Vio, R., Rebusco, P., Andreani, P., Madsen, H., \& Overgaard, R. V. 2006, A\&A, 452,383

Zanotti, O., Font, J. A., Rezzolla, L., \& Montero, P. J. 2005, MNRAS, 356, 1371 